\section{CLOSER, WISER, STRONGER: SETTING UP A PEER-LED REFLECTIVE PRACTICE GROUP FOR PALLIATIVE MEDICINE SPECIALTY REGISTRARS}

Robert Brodrick, Abi Ponnampalam. Arthur Rank Hospice Charity, Farleigh Hospice

10.1136/spcare-2020-PCC.31

Background Palliative care clinicians are routinely exposed to intense distress. Maslach Burnout Inventory data suggests this is a major workforce issue and correlates with increased risk of emotional exhaustion. Interventions to increase self-awareness may reduce this risk; the GMC recommends group reflection as it can lead to improved patient care. Balint groups are an approach to reflective practice that examine the doctor-patient relationship. A recent review concluded that they should form a key component of palliative medical training.

Methods We established a confidential, co-created, peer-led reflective practice group based on Balint principles for palliative medicine specialty registrars. To our knowledge, this is a UK first. It was designed to help develop specific competencies in the current Specialty Training Curriculum such as Self Awareness (5.1), The Doctor-Patient Relationship (5.3) and Learning and Self-Development (10.1)

Whilst traditional Balint groups are facilitated by an experienced psychotherapist, we used a rotating peer facilitator to promote group work skills, with the advantage of being cost neutral. 14 specialty registrars met together for an hour-long reflective practice group each month during seven consecutive regional training days. We surveyed members' views anonymously on the effects of group participation.

Results $100 \%$ (14/14) felt the group was an effective use of training time and were keen for the group to continue. 93\% (13/14) thought it enhanced their ability to adapt to workrelated challenges. $100 \%(14 / 14)$ valued the opportunity to learn from others' experience, 93\% found it enhanced peer support (13/14), 79\% (11/14) felt less isolated as a result and $71 \%(10 / 14)$ thought the group helped develop self-awareness and insight. The most frequent words used to thematically describe the group were: supportive, valuable, safe, reassuring, interesting and useful.

Conclusions Starting a peer-led reflective practice group is an efficient, effective and economic method of enhancing higher specialty training in palliative medicine.

\section{ONE OPIOID CONVERSION WORKBOOK, THREE SETTINGS: COMMUNITY, HOSPICES, HOSPITALS}

Robert Brodrick, Sylvia Reid, Sarah Mollart. Norfolk and Norwich University Hospital, Sue Ryder Thorpe Hall Hospice, St Nicholas Hospice Care, West Suffolk Hospital

10.1136/spcare-2020-PCC.32

Background Despite a near absence of rigorous training programmes, safely converting between strong opioids is a key skill for palliative care clinicians, regardless of setting.

To address this deficit, we developed the Opioid Conversion Workbook (OCW), an educational intervention to teach complex conversion calculations between morphine (oral and subcutaneous), oxycodone (oral and subcutaneous), transdermal buprenorphine, transdermal fentanyl and subcutaneous alfentanil.

Aims To assess whether the OCW produced improvements in opioid conversion ability in different professions, clinical settings and experience levels, and whether these improvements were sustained.

Methods Participants were selected across the East of England from the palliative care teams of a university teaching hospital, a district general hospital, two community clinical nurse specialist (CNS) teams, and two specialist palliative care inpatient units (IPU).

Each participant answered 17 clinically relevant calculation questions as a baseline assessment under exam conditions. They then completed the OCW over 6 weeks, before taking a different but structurally symmetrical 17 question final assessment. One year after completing the OCW, a proportion of participants took an ability retention assessment under exam conditions.

Results 141 clinical staff took part: 64 hospice IPU nurses, 35 hospice doctors, 17 hospital CNS, 2 hospital doctors, 18 community CNS, and 5 clinical managers. The length of time working in palliative care ranged from 1 week to 25 years.

The median baseline assessment score was 34\% (5.79/17; range 0 to 17). Following completion of the OCW, the median final assessment score was 99.8\% (16.96/17; range 15 to 17$)$.

12 months later, 23 staff took the ability retention assessment. The mean score was $91.8 \%$ (15.6/17; range 8 to 17$)$. Conclusions The OCW generated significant improvements in opioid conversion ability for a wide range of palliative care staff, in a variety of clinical settings. Most of this improvement was sustained, suggesting long-term skill retention.

\section{COMPETENCY BASED PALLIATIVE CARE EDUCATION IN ACUTE HOSPITAL}

Lynne Cannell, Anita Roberts, Sarika Hanchanale. Royal Liverpool Hospital

\subsection{6/spcare-2020-PCC.33}

Background All Healthcare professionals including nurses should be competent to provide the best end of life care. We developed Professional Development in Palliative and End of Life Care Programme to enhance learning and teaching to registered nurses and allied healthcare professionals. The aim of the programme is to provide high quality evidence-based end of life care and maintain the Care Quality Commission (CQC) rating of "Outstanding".

Methods This in-house programme uses the End of Life Care core skills education and training framework, developed by Health Education England. Programme consists 5 half-day and 1 full day study sessions for the theoretical component of the course and half-day to shadow Palliative Care Clinical Nurse Specialist mentor. The practical elements are work-based and are guided by a competency portfolio and reflective practice. Evaluations were completed pre- and post-teaching assessing knowledge, skills and confidence on $0-5$ scale for each session.

Results Total 34 attendees attended from September 2018-June 2019 in 3 cohorts. Attendees found every session helpful, interesting and informative, 'Pain management' session was most informative of all. There was increase in knowledge, skills and confidence after each session. Average increase in knowledge in psychological issues from 2.61 to 3.85 , principles of pain management from 2.23 to 4.26. Average increase in skills in palliative care emergencies was from 2.76 to 3.88 , ethical dilemmas was from 2.67 to 3.20 . Average increase in 